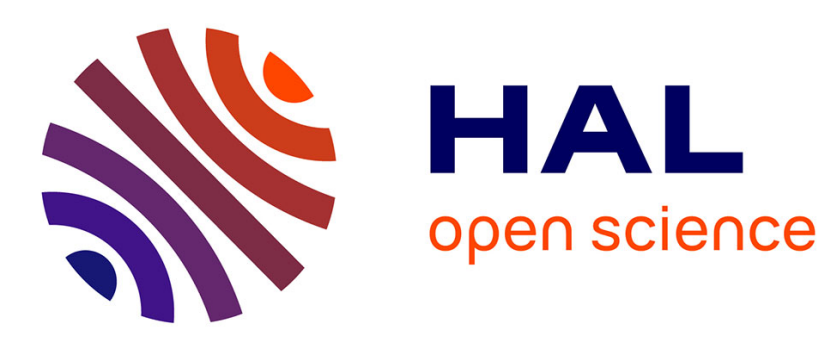

\title{
The role of thermophoresis on aluminum oxide lobe formation
}

Stany Gallier, Alexandre Braconnier, Franck Godfroy, Fabien Halter, Christian Chauveau

\section{- To cite this version:}

Stany Gallier, Alexandre Braconnier, Franck Godfroy, Fabien Halter, Christian Chauveau. The role of thermophoresis on aluminum oxide lobe formation. Combustion and Flame, 2021, 228, pp.142-153. 10.1016/j.combustflame.2021.01.039 . hal-03135480

\section{HAL Id: hal-03135480 https://hal.science/hal-03135480}

Submitted on 9 Feb 2021

HAL is a multi-disciplinary open access archive for the deposit and dissemination of scientific research documents, whether they are published or not. The documents may come from teaching and research institutions in France or abroad, or from public or private research centers.
L'archive ouverte pluridisciplinaire HAL, est destinée au dépôt et à la diffusion de documents scientifiques de niveau recherche, publiés ou non, émanant des établissements d'enseignement et de recherche français ou étrangers, des laboratoires publics ou privés. 


\title{
The role of thermophoresis on aluminum oxide lobe formation
}

\author{
Stany GALLIER ${ }^{\mathrm{a}, *}$, Alexandre BRACONNIER ${ }^{\mathrm{a}, \mathrm{b}, * *}$, Franck GODFROY $^{\mathrm{a}}$, Fabien HALTER ${ }^{\mathrm{b}}$, Christian \\ CHAUVEAU ${ }^{\mathrm{b}}$ \\ ${ }^{a}$ ArianeGroup, Le Bouchet Research Center, 91710 Vert-le-Petit, France \\ ${ }^{b}$ CNRS-ICARE, University of Orléans, 45000 Orléans, France
}

\begin{abstract}
This work studies the influence of phoretic motion (thermophoresis and diffusiophoresis) of fine alumina particles ("smoke") produced during the combustion of aluminum. Direct numerical simulations on a single aluminum droplet burning in a quiescent environment suggest that thermophoresis is the main mechanism driving smoke back to the aluminum surface, hence a major contributor to the oxide lobe development. The presence of this lobe is found to distort the flowfield, which favors hot and smoke-rich regions closer to the lobe, thereby enhancing thermophoresis. This combination of aerodynamic and thermophoretic effects leads to a mass rate of deposited smoke which is consistent with experimental data. A simplified model, deduced from simulation results, is able to predict the size of the final oxide residue in good agreement with measurements. This study supports that aluminum oxide present on the burning aluminum particle is largely due to material formed in the flame, subsequently desposited by thermophoresis.
\end{abstract}

Keywords:

Aluminum combustion, Oxide lobe, Solid propulsion, Thermophoresis

\section{Introduction}

Aluminum particles are widely used for space and military applications since aluminum combustion significantly enhances the performance of solid rocket motors. Aluminum has also received a growing interest in fuel cells [1] or novel power generation concepts [2, 3] as it provides a carbon-free energy. However aluminum combustion is complex and, despite decades of fundamental studies, not completely understood so far.

It is generally accepted that large aluminum particles (i.e. $\gtrsim 10-20 \mu \mathrm{m}$ ) burn in the vapor-phase through a diffusion flame [4]. Combustion of aluminum produces aluminum oxide (alumina, $\mathrm{Al}_{2} \mathrm{O}_{3}$ ) as the major product. It is predominantly present as fine particlescommonly referred to as oxide smoke with typical size $d_{o x} \approx 1 \mu \mathrm{m}$-in the detached flame around the burning aluminum particle. In addition, aluminum oxide is also present on the burning particle as a distinct cap, or lobe, because liquid aluminum and its oxide are nonmiscible. When aluminum is fully consumed, this lobe remains as an oxide particle, or residue. This residue

\footnotetext{
*Corresponding author: stany.gallier@ariane.group ** Present address: MBDA, Route d'Issoudun, 18020 Bourges
}

can be as large as the initial aluminum particle [5].

The presence of an oxide lobe on the burning aluminum particle decreases the available surface for aluminum evaporation and is sometimes deemed to be responsible from the deviation from the expected $d^{2}$ law $[4,6,7]$. Note that the existence of this lobe is a distinctive feature of aluminum combustion compared to liquid hydrocarbon fuels. In the field of solid rockets, oxide residues in the combustion chamber can significantly contribute to two-phase performance losses when ejected through the nozzle [8]. Recent studies have also pointed out that burning of aluminum could trigger thermoacoustic instabilities in solid rocket motors $[9,10]$ and that the size of this oxide residue could play a significant role [11].

The diameter of the final oxide residue $d_{\text {res }}$ is usually linearly related to the size of the initial aluminum particle $d_{A l}$ by $d_{r e s}=\beta d_{A l}$. The proportionality factor $\beta$ has scattered values from the literature: Salita [8] compiled various experimental results and found values in the range $0.5 \sim 0.8$. Turns et al. [12] experimentally deduced $\beta$ between 0.6 and 0.8 . Glotov and Zhukov [13] measured values in the range $0.5 \sim 0.8$ depending on diameters and oxidizers while Zenin et al. [14] found $\beta \approx$ 0.8 . Lower values $\beta \approx 0.2$ were also attested in quench 
bomb measurements on aluminized solid propellants, with a strong effect of the pressurant [15]. Similarly, experimental observations suggest that the lobe is more massive when nitrogen is used as a diluent compared to argon or helium $[16,17,18]$. Other experiments show that the rate of oxide accumulation grows for larger aluminum particles and lower oxygen content [19].

A clear physical mechanism for oxide lobe formation is still missing. Some authors propose that there could be some deposition of smoke on the particle but without any further detailed mechanism [20, 21, 22]. On the other hand, Dreizin [17] expects this deposition of smoke to be negligible due to the strong outward flow (Stefan flow) on particle surface. He therefore advocates a diffusion of gaseous aluminum suboxides to the particle surface which could saturate an oxygen-rich phase with subsequent transformation into stoichiometric oxide. It seems also that oxide lobes are formed through material deposited from the flame rather through an internal transition [5]. King [7] proposed a simplified model assuming that $\mathrm{AlO}$ produced in the flame diffuses back and undergoes collision-limited reaction with the surface to form liquid $\mathrm{Al}_{2} \mathrm{O}_{3}$. His model was applied to the combustion of a $100 \mu \mathrm{m}$ aluminum particle burning in a solid rocket and gave $\beta \approx 0.7$. Glorian et al. [23, 24] developed a surface reaction mechanism using ab initio computations and used it for combustion simulations of a burning aluminum droplet. They showed that oxygen-rich gaseous species diffuse to the surface, then adsorbate and react to form liquid alumina $\mathrm{Al}_{2} \mathrm{O}_{3}$ on aluminum burning surface. They showed that $\beta$ was much dependent on particle size and nature of oxidizer and, for a $d_{A l}=100 \mu \mathrm{m}$ particle in air, computed $\beta=0.26$, which is significant but still lower than most experiments. This suggests that heterogeneous reactions are a possible route for alumina production directly on aluminum surface but might not be the only mechanism.

Detailed direct numerical simulations on a single burning droplet are available in the literature $[4,25$, 26, 27, 28, 29, 24] and they all consider that smoke produced in the flame diffuses through a Fick's law, with fume diffusion coefficients identical to gaseous species. This certainly oversimplifies the physics although it has provided reliable estimates of deposition rates [30]. The oxide lobe has been accounted for in some simulations $[26,25]$-yet simplified as a spherical cap. Although the lobe seems to induce a distortion of the species/temperature profiles, there are no detailed results whether the presence of the lobe could promote smoke deposition.

This literature survey does not clearly identify a unique and well-accepted mechanism for oxide lobe formation. Production of alumina on particle surface due to chemical reactions from gaseous suboxides diffusing from the flame is commonly accepted although it may not completely explain experimental results. The possibility of alumina smoke diffusing back onto the aluminum surface remains an additional plausible mechanism but this requires further inspection.

In this work, we investigate the role of phoretic motion as a possible deposition mechanism of smoke from the flame to the aluminum particle surface. Unlike previous works, there is here no need to postulate a Fickian diffusion for smoke particles. This paper starts in Section 2 with some experimental observations that highlight the role of the oxide lobe during combustion. In Section 3, we discuss the possibility of phoretic motion for smoke as well as we deal with the exact geometry expected for a composite aluminum droplet with its oxide lobe. The numerical model is presented in Section 4 before moving to our results in Section 5 showing how thermophoresis, augmented by some aerodynamic effects due to the presence of the lobe, can involve the formation of oxide lobe during aluminum combustion. This study is restricted to oxygen-containing environments so that only oxide is considered. Carbon-rich or nitrogen-rich environments can produce carbides or nitrides [31, 32, 33] but this will therefore not be considered in this study.

\section{Experimental observations}

\subsection{Effect of the oxide lobe during combustion}

The present study was to some extent motivated by some of our recent experimental observations conducted in our electrostatic levitator $[34,35,16]$. In this set-up, a single aluminum droplet is levitated by electrostatic forces, ignited by a $\mathrm{CO}_{2}$ laser beam, and burns in a controlled environment, allowing a large spectrum of burning conditions, such as pressure or oxidizers. High temporal and spatial resolution can be obtained, up to $40000 \mathrm{fps}$ and $2.5 \mu \mathrm{m} / \mathrm{pixel}$, using a high-speed camera. We do not describe the set-up in the frame of this paper and the interested reader may refer to the previously cited references.

An interesting sequence, taken from Braconnier [36], is presented in Fig. 1 and was obtained for a $d_{A l}=95 \mu \mathrm{m}$ particle burning in a $\mathrm{CO}_{2} / \mathrm{O}_{2}=(40 / 60)$ mixture at $p=1$ atm. Images are taken from a high-speed video and are typically separated by $0.5 \mathrm{~ms}$. Image (1) is taken right after ignition and a large cloud of alumina particles is emitted and progressively evacuated by the bottom in 
the subsequent images. The oxide lobe-visible as a bright white spot-is clearly apparent from the beginning but may occasionally disappear as the particle rotates. A clear motion of smoke then becomes visible on the video, indicated by red arrows in image (5) et seq., and this strengthens with time. The overall smoke motion is unambiguously directed towards the oxide lobe. In images (7) and (8), the motion of smoke can even be noticed in the flame at the opposite side of the lobe (small arrows), indicating a large-scale transfer across the whole flame. During this stage, the lobe grows significantly and at some point, the particle undergoes a rapid spinning in image (10).

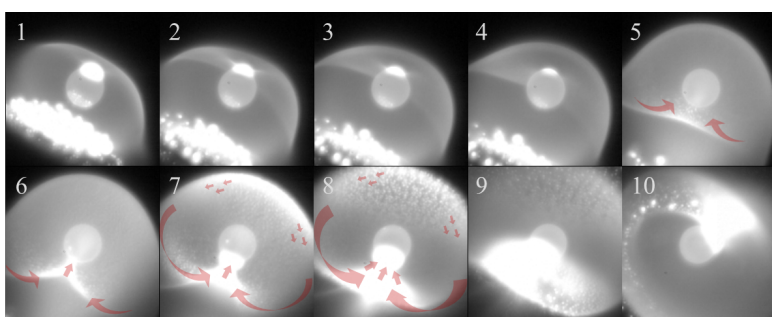

Figure 1: Sequence of a $d_{A l}=95 \mu \mathrm{m}$ particle burning in a $40 \% \mathrm{CO}_{2} / 60 \% \mathrm{O}_{2}$ mixture at $p=1 \mathrm{~atm}$. [36].

This observation calls for a significant motion of smoke towards the particle, mostly through the lobe. Among all experiments conducted, this behavior is not systematically noticed in such a clear manner. It seems however that it is promoted when a significant oxide lobe is present at the early stages of burning, which was often the case in $\mathrm{CO}_{2}$-rich environments. Nonetheless, this effect still persists in many other gases, including air. As an example, Fig. 2 shows a relatively similar sequence in a $\mathrm{N}_{2} / \mathrm{O}_{2}$ mixture. A small lobe is visible as a bright point on the particle in image (1) and is subsequently hidden in image (2) as the particle rotates. The onset of smoke motion starts from image (3) and is quite clear in image (4) where the lobe has already significantly grown.

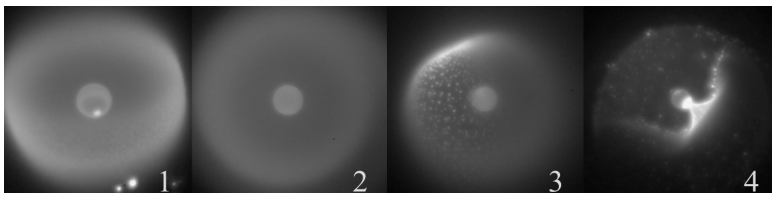

Figure 2: Sequence of a $d_{A l}=93 \mu \mathrm{m}$ particle burning in a $40 \% \mathrm{~N}_{2} / 60 \% \mathrm{O}_{2}$ mixture at $p=1$ atm. [36].

The resulting increase in smoke concentration in the vicinity of the lobe gives rise to a brighter zone in the flame region. This led Dreizin [17] to suggest the occurrence of a so-called asymmetric flame regime. We do believe that this "asymmetric" regime is actually just related to the rapid build-up of smoke above the lobe. This symmetric/asymmetric transition was studied recently by the present authors [16] and we found that the nature of gas is of primary importance. The fraction $\tau$ of the burning time spent in the symmetric regime is in the range $0.9 \sim 1$ (i.e., no asymmetric regime) for $\mathrm{O}_{2} / \mathrm{He}$ and $\mathrm{O}_{2} / \mathrm{Ar}$ mixtures at large dilution ratio $\left(\mathrm{O}_{2}<\right.$ $40 \%$ ). Conversely, it was typically $0.5 \sim 0.6$ when $\mathrm{N}_{2}$ was used as a diluent (irrespective of oxidizer content) and about $\tau=0.4$ in pure $\mathrm{O}_{2}$ or pure $\mathrm{CO}_{2}$. Those results therefore suggest that the development of the lobe (and subsequent transition to asymmetric regime) is strongly related to the nature of gas.

We end this section by a last and highly instructive sequence taken in a $\mathrm{CO}_{2} / \mathrm{O}_{2}$ mixture and presented in Fig. 3. The first images show a rather hectic ignition with many large alumina droplets dispersed in the flame. They progressively coalesce and are finally evacuated from the flame region from image (6). It seems that during those events, the oxide lobe may have been expelled as well. Thereafter, we observe a mild and steady combustion until complete burning. There is no visible lobe in the videos, nor asymmetric flame, and the particle is fully burnt out without any jetting and spinninga rather unusual case in such environments. This unequivocally shows that when no initial lobe is present, then no lobe grows. This definitely spotlights the role of the initial lobe and possibly suggests that surface reactions during combustion are possibly too weak to induce a significant oxide production.

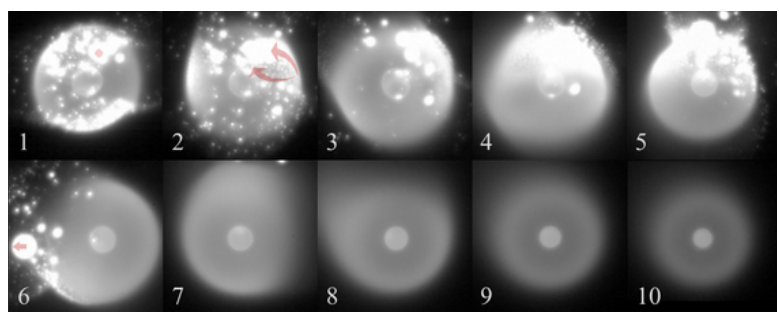

Figure 3: Sequence of a $d_{A l}=70 \mu \mathrm{m}$ particle burning in a $60 \% \mathrm{CO}_{2} / 40 \% \mathrm{O}_{2}$ mixture at $p=1 \mathrm{~atm}$. [36].

Let us here quickly summarize our observations. High-speed videos of a single burning aluminum particle support the idea that the initial presence of a lobe triggers an intense motion of smoke towards this lobe that, in turn, feeds it and makes it grow. The role of this initial lobe is substantiated by an experiment when com- 
bustion proceeds without such smoke motion when the initial lobe is missing. We are aware that more analysis is needed (including the effect of gas nature, pressure, etc.) before a definite and general conclusion can be drawn but we however believe that this puts forward a primary role of the initial oxide lobe on the underlying physical mechanism. Those observations have nevertheless fostered the following numerical works in order to provide further illumination.

\section{About smoke motion and lobe shape}

Before moving to numerical modeling, we here discuss two important aspects needed for the forthcoming simulations. The first one is linked to the possible mechanisms responsible for small particle motion and the second is the exact shape of an aluminum particle with its oxide lobe, which is needed to define the geometry in simulations.

\subsection{Mechanisms of oxide smoke motion}

A basic-and commonly accepted-assumption is that alumina $\mathrm{Al}_{2} \mathrm{O}_{3}$ produced during the combustion of aluminum takes the form of small particles ("smoke"), which are liquid at the temperatures of interest (melting temperature of alumina is about $2330 \mathrm{~K}$ ). There is actually no consensus about whether alumina $\mathrm{Al}_{2} \mathrm{O}_{3}$ could have a stable gas phase, see Savel'ev and Starik [37] for a discussion. In their work, those latter authors describe the formation of $\left(\mathrm{Al}_{2} \mathrm{O}_{3}\right)_{n}$ clusters as a possible mechanism of aluminum oxide nucleation. In present work however, we discard any gas-phase $\mathrm{Al}_{2} \mathrm{O}_{3}$ or clusters thereof and assume that alumina is completely represented as small liquid particles. The diameter $d_{o x}$ of this smoke phase is typically about $1 \mu \mathrm{m}$ and reported values in the literature give a mean mass diameter of 0.8 $\mu \mathrm{m}$ [38], $1.5 \mu \mathrm{m}$ [22], or the range 0.2 2 $\mu \mathrm{m}$ [21]. Salita [8] compiled values from the literature and suggests a mean mass diameter value of $1 \mu \mathrm{m}$. Therefore the value $d_{o x}=1 \mu \mathrm{m}$ is taken in this work as a relevant average value. In reality, smoke size may possibly range between nano to several micrometers but detailed data are lacking. Therefore, for the sake of simplicity, a single smoke size $d_{o x}=1 \mu \mathrm{m}$ is chosen. Some parametric simulations discussed in Sec. 5 show that smoke size is a second-order parameter.

An assumption is that alumina is only under a liquid phase. This rules out any possibility of motion back to the aluminum particle surface due to the usual Fickian diffusion, which is only active at the molecular scale, hence gas-phase. As already addressed in the introduction, computational studies from the literature assume for simplicity that alumina may diffuse with classical Fick's law with diffusion parameters taken to that of the (hypothetical) $\mathrm{Al}_{2} \mathrm{O}_{3}$ gas species. Here, we aim at departing from this assumption-which we believe, is deprived of any physical grounds-and consider liquid phase alumina. Small particles may therefore be subjected to convection (i.e., carried by the gas flow), Brownian motion, as well as phoretic motion, i.e. motion of particles under gradients of an external thermodynamic variable. Among the various phoretic motions, we only retain thermophoresis (due to temperature gradients) and diffusiophoresis (due to gas concentration gradients) as the most important mechanisms. Turbophoresis (due to turbulence) is neglected because of very low Reynolds numbers. We moreover assume that electrophoresis (due to electric charges) is weak, because of intense thermoionic emission at the high temperatures of smoke in the flame. This seems supported by experiments in our electrodynamic levitator $[34,35,16]$ where no deformations of the flame due to the imposed electric field were ever observed.

Thermophoresis induces the displacement of suspended particles under the influence of an applied thermal gradient owing to the difference in the momentum transfer to the particle between gas molecules with a high thermal velocity and those with a low thermal velocity. Since it is proportional to the temperature gradient $\nabla T$, thermophoresis is believed to be significant for smoke, between the detached flame and the burning aluminum surface, where temperature gradient is large, typically $\nabla T \sim 10^{7} \mathrm{~K} / \mathrm{m}$. The thermophoretic velocity $\mathbf{v}_{t h}$ is given by [39] as

$$
\mathbf{v}_{t h}=-K_{t h} \frac{\mu}{\rho} \frac{\nabla T}{T}
$$

with

$$
K_{t h}=2.294 C_{c} \frac{\lambda^{*}+2.18 K n}{(1+3.44 K n)\left(1+2 \lambda^{*}+4.36 K n\right)}
$$

and the Cunningham coefficient

$$
C_{c}=1+K n(1.26+0.4 \exp (-1.1 / K n))
$$

where $\rho$ is the gas density, $\mu$ the gas dynamic viscosity, $T$ the temperature and $\lambda^{*}$ the ratio between the thermal conductivity of gas and smoke particles $\lambda^{*}=\lambda_{g} / \lambda_{o x}$. A value $\lambda_{o x}=7 \mathrm{~W} / \mathrm{m} / \mathrm{K}$ is taken for alumina at high temperatures. In flame conditions at atmospheric pressure, the molecular mean free path $l$ is typically about $0.5 \mu \mathrm{m}$, so that the Knudsen number $K n=2 l / d_{o x} \approx 1$, suggesting a transitional flow at the smoke size. 
Diffusiophoresis occurs under a gradient of chemical concentration. It has received less attention than thermophoresis [40, 41, 42] and the most studied case is the diffusiophoresis of particles in a binary gas mixture where the diffusiophoretic velocity $\mathbf{v}_{d}$ reads [40, 42]

$$
\mathbf{v}_{d}=-\frac{\mathcal{M}_{1}^{1 / 2}-\mathcal{M}_{2}^{1 / 2}}{X_{1} \mathcal{M}_{1}^{1 / 2}+X_{2} \mathcal{M}_{2}^{1 / 2}} \mathcal{D}_{1-2} \nabla X_{1}
$$

where $X$ notes the molar fraction, $\mathcal{M}$ the molar mass, and $\mathcal{D}_{1-2}$ the binary diffusion coefficient of species 1 in 2. Extension to multicomponent gas is scarce and an approximate form proposed by Whitmore [43] will be chosen here as

$$
\mathbf{v}_{d}=-\frac{\sum_{k} \mathcal{M}_{k}^{1 / 2}}{\sum_{k} X_{k} \mathcal{M}_{k}^{1 / 2}} \mathcal{D}_{k} \boldsymbol{\nabla} X_{k}
$$

with $\mathcal{D}_{k}$ the diffusion coefficient of (gas-phase) species $k$ in the mixture.

\subsection{Particle and lobe shape}

In the following, we theoretically describe the shape of an aluminum particle with its oxide lobe, which will define the exact geometry needed for simulations. We believe this is worth describing since it has been scarcely addressed in the open literature-only by Babuk et al. [20] as far as we are aware. Taking the exact theoretical shape is expected to be more relevant than the spherical cap approximation used in previous works $[26,25]$. As far as we are aware, there are no reported simulations with the exact lobe shape. Because aluminum and alumina are immiscible, alumina produced or deposited on the particle will build up as a spherical lobe. Alumina is expected to be carried to the lobe by the internal recirculation of liquid aluminum inside the droplet (Hill's vortex). This exact shape can be obtained using the equilibrium of the triple line $\mathrm{T}$ (see Fig. 4) between surrounding gas, aluminum $(A l)$ and alumina $(O x)$. Noting $\sigma$ the surface tension, this reads

$$
\begin{aligned}
\sigma_{A l-o x}+\sigma_{o x} \cos \theta_{2}+\sigma_{A l} \cos \theta_{1} & =0 \\
\sigma_{A l-o x} \cos \theta_{2}+\sigma_{o x}+\sigma_{A l} \cos \left(\theta_{1}+\theta_{2}\right) & =0 \\
\sigma_{A l-o x} \cos \theta_{1}+\sigma_{o x} \cos \left(\theta_{1}+\theta_{2}\right)+\sigma_{A l} & =0
\end{aligned}
$$

where angles $\theta_{i}$ are taken as in the figure. They can be expressed in terms of (known) surface tensions by

$$
\begin{aligned}
& \cos \theta_{1}=\frac{\sigma_{o x}^{2}-\sigma_{A l-o x}^{2}-\sigma_{A l}^{2}}{2 \sigma_{A l-o x} . \sigma_{A l}} \\
& \cos \theta_{2}=\frac{\sigma_{A l}^{2}-\sigma_{A l-o x}^{2}-\sigma_{o x}^{2}}{2 \sigma_{A l-o x} . \sigma_{o x}}
\end{aligned}
$$

The angle $\alpha$ is introduced to define the position of the triple line (see figure), from which we get the relation between aluminum radius $R_{A l}$, lobe radius $R_{o x}$ and interface radius $R_{A l-o x}$ by

$$
R_{A l} \cos \alpha=R_{o x} \cos (\alpha-\delta)=R_{A l-o x} \cos \left(\theta_{1}-\alpha\right)
$$

where we have noted $\delta=\theta_{1}+\theta_{2}-\pi$ for convenience. The next step consists in evaluating the volume $V_{A l}$ and $V_{o x}$ of aluminum and alumina, which is simple geometry, and finally yields

$$
\begin{gathered}
V_{A l}=\frac{\pi}{3} R_{A l}^{3}\left[(1+\sin \alpha)^{2}(2-\sin \alpha)-\frac{\cos ^{3} \alpha}{\cos ^{3}\left(\theta_{1}-\alpha\right)} .\right. \\
\left.\left(2+\sin \left(\theta_{1}-\alpha\right)\right)\left(1-\sin \left(\theta_{1}-\alpha\right)\right)^{2}\right] \quad(12) \\
V_{o x}=\frac{\pi}{3} R_{A l}^{3}\left[\frac{\cos ^{3} \alpha}{\cos ^{3}(\alpha-\delta)}(1-\sin (\alpha-\delta))^{2}(2+\sin (\alpha-\delta))+\right. \\
\left.\frac{\cos ^{3} \alpha}{\cos ^{3}\left(\theta_{1}-\alpha\right)}\left(2+\sin \left(\theta_{1}-\alpha\right)\right)\left(1-\sin \left(\theta_{1}-\alpha\right)\right)^{2}\right]
\end{gathered}
$$

Now the procedure is the following. Since surface tensions $\sigma_{i}$ are given input data, angles $\theta_{i}$ and $\delta$ can be estimated through Eqs. (9)-(10). It can be noted that the ratio between $V_{o x}$ and $V_{A l}$ depends only on $\alpha$ $V_{o x} / V_{A l}=f(\alpha)$, so that for a given volume ratio we solve this non-linear equation for $\alpha$. Then, radii are deduced from Eq. (11) and the distance between sphere centers by $\left\|O_{A l} O_{o x}\right\|=\left(R_{A l}^{2}+R_{o x}^{2}-2 R_{A l} R_{o x} \cos \delta\right)^{1 / 2}$. The interface radius $R_{A l-o x}$ is found to be negative (such as in the schematic of Fig. 4) for low alumina content and positive (oriented the other way) for larger lobes. The interface becomes plane for angle $\alpha=\theta_{1}-\pi / 2$.

The surfaces of the aluminum particle $S_{A l}$ and oxide lobe $S_{o x}$ are obtained by

$$
\begin{aligned}
& S_{A l}=2 \pi R_{A l}^{2}(1+\sin \alpha) \\
& S_{o x}=2 \pi R_{o x}^{2}(1-\sin (\alpha-\delta))
\end{aligned}
$$

Surface tensions, as a function of temperature $T$, need to be prescribed as well as density since mass $m_{i}$ are generally known rather than volumes $V_{i}$ in order to solve $V_{o x} / V_{A l}=\rho_{A l} m_{o x} / \rho_{o x} m_{A l}=f(\alpha)$. Relations used are taken from the literature and are the following (units: $\mathrm{K}, \mathrm{kg} / \mathrm{m}^{3}, \mathrm{~N} / \mathrm{m}$ )

$$
\begin{aligned}
\rho_{A l} & =3226-0.604 T \\
\rho_{o x} & =3056-0.97(T-2327) \\
\sigma_{A l} & =1.267-2.6 .10^{-4}(T-933) \\
\sigma_{o x} & =0.66-6.10^{-5}(T-2327) \\
\sigma_{A l-o x} & =0.687-1.6 .10^{-4}(T-933)
\end{aligned}
$$




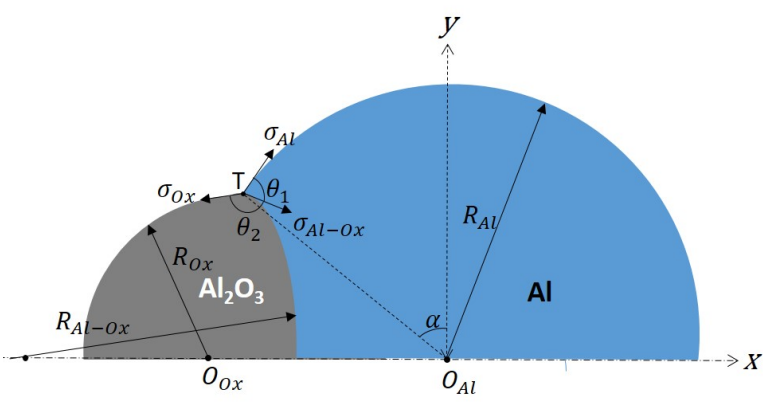

Figure 4: Aluminum droplet (blue) with its oxide lobe (grey).

Droplet temperature $T$ is known from the simulations. Let us note $f_{o x}$ the mass fraction of alumina in this binary system $f_{o x}=m_{o x} /\left(m_{A l}+m_{o x}\right)$. Figure 5 presents examples of geometries computed for four different alumina mass fractions $f_{o x}=0.1,0.3,0.5$, and 0.7 with constant aluminum diameter $d_{A l}=2 R_{A l}=70 \mu \mathrm{m}$. They will be used in the computations in Sec. 5. For those four values, the fraction of aluminum particle surface covered by the lobe $\chi_{o x}=0.5(1-\sin \alpha)$ is respectively 0.09 , $0.21,0.33$, and 0.46 (a)

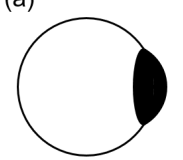

(c)

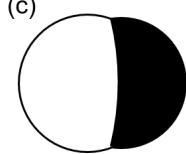

(b)

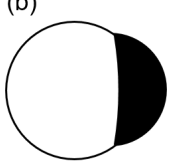

(d)

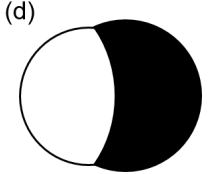

Figure 5: Aluminum (white)/alumina (black) droplet geometry for four alumina mass fractions $f_{o x}=0.1(\mathrm{a}), 0.3(\mathrm{~b}), 0.5(\mathrm{c})$, and $0.7(\mathrm{~d})$.

\section{Numerical model}

This work considers direct numerical simulations on a single burning aluminum particle. We are interested in steady combustion - and not ignition-and we therefore assume that the thin passivating alumina layer is already molten. Aluminum particle, as well as its oxide lobe, are assumed to be isothermal due to small Biot numbers. The flow inside the droplet is not considered and only the exterior domain (gas phase) is modeled. This work essentially focuses on micrometer-scale aluminium particles, and diameters $d_{A l}$ investigated lie the range $20 \sim 400 \mu \mathrm{m}$. Hence, the Knudsen number at the particle scale $K n=2 l / d_{A l}$ is about 0.01 , which legitimates considering the usual Navier-Stokes equations without any slip corrections.

\subsection{Governing equations}

Accounting for spherical symmetry, the twodimensional axisymmetric reactive Navier-Stokes equations are solved around the particle. Conservation of mass, momentum, energy and species can be written as

$$
\frac{\partial \mathbf{U}}{\partial t}+\frac{\partial \mathbf{F}_{c}}{\partial x}+\frac{\partial \mathbf{G}_{c}}{\partial y}=\frac{\partial \mathbf{F}_{d}}{\partial x}+\frac{\partial \mathbf{G}_{d}}{\partial y}+\mathbf{S}
$$

where $\mathbf{U}$ is the vector of conservative variables whereas $\mathbf{F}_{c}$ and $\mathbf{G}_{c}$ are the convective fluxes in the $x$ and $y$ direction respectively (see Fig. 4) :

$\mathbf{U}=y\left[\begin{array}{c}\rho \\ \rho u \\ \rho v \\ \rho E \\ \rho Y_{k}\end{array}\right]$

$$
\mathbf{F}_{c}=y\left[\begin{array}{c}
\rho u \\
\rho u^{2}+p \\
\rho u v \\
\rho u E+p u \\
\rho u Y_{k}
\end{array}\right]
$$$$
\mathbf{G}_{c}=y\left[\begin{array}{c}
\rho v \\
\rho u v \\
\rho v^{2}+p \\
\rho v E+p v \\
\rho v Y_{k}
\end{array}\right]
$$

where $\rho$ is the density, $u$ and $v$ the gas velocity in $x$ and $y$ directions, $p$ the pressure, $E$ the total energy and $Y_{k}$ the mass fraction of the $k^{\text {th }}$ species (this includes both gasphase species and smoke phase, see forthcoming section for details). The diffusive fluxes $\mathbf{F}_{d}$ and $\mathbf{G}_{d}$ are given by

$\mathbf{F}_{d}=y\left[\begin{array}{c}0 \\ \tau_{x x} \\ \tau_{x y} \\ u \tau_{x x}+v \tau_{x y}-q_{x} \\ -J_{k, x}\end{array}\right] \quad \mathbf{G}_{d}=y\left[\begin{array}{c}0 \\ \tau_{x y} \\ \tau_{y y} \\ u \tau_{x y}+v \tau_{y y}-q_{y} \\ -J_{k, y}\end{array}\right]$

The shear stress tensor $\tau$ is given as

$$
\begin{aligned}
\tau_{x x} & =\mu\left(\frac{4}{3} \frac{\partial u}{\partial x}-\frac{2}{3} \frac{\partial v}{\partial y}\right) \\
\tau_{x y} & =\mu\left(\frac{\partial u}{\partial y}+\frac{\partial v}{\partial x}\right) \\
\tau_{y y} & =\mu\left(\frac{4}{3} \frac{\partial v}{\partial y}-\frac{2}{3} \frac{\partial u}{\partial x}\right)
\end{aligned}
$$

where $\mu$ is the gas mixture viscosity. The heat flux $\mathbf{q}$ and diffusion flux $\mathbf{J}_{k}$ for the $k^{\text {th }}$ species are expressed as

$$
\begin{array}{cc}
q_{x}=-\lambda \frac{\partial T}{\partial x} & q_{y}=-\lambda \frac{\partial T}{\partial y} \\
J_{k, x}=-\rho \mathcal{D}_{k} \frac{\partial Y_{k}}{\partial x} & J_{k, y}=-\rho \mathcal{D}_{k} \frac{\partial Y_{k}}{\partial y}
\end{array}
$$


where $\lambda$ is the gas mixture thermal conductivity and $\mathcal{D}_{k}$ is the gas mass diffusion coefficient of species $k$ in the mixture. In Eq. (16), the source term is expressed as

$$
\mathbf{S}=\left[0,0, p-\tau_{\theta}, 0, \rho y \dot{\omega}_{k}^{g}\right]^{t}
$$

where $\dot{\omega}_{k}^{g}$ is the mass production rate for the $k^{\text {th }}$ species from gas-phase reactions. Note that due to the axisymmetric formulation, an additional source term on the $y$ momentum equation arises with

$$
\tau_{\theta}=\mu\left(2 \frac{v}{y}-\frac{2}{3}\left(\frac{\partial u}{\partial x}+\frac{\partial v}{\partial y}\right)\right)
$$

The source term in the energy equation is zero because the reference energy is already included in the total energy $E$. Radiation has been neglected here since its contribution is believed to be limited [47]. Finally, a perfect gas law is used with $p=\rho R T / \mathcal{M}$ with $\mathcal{M}$ the average molecular mass.

Transport coefficients are computed for each species using standard kinetic theory. Mixture laws are then used to obtain mixture viscosity, conductivity, and mass diffusivity. Mixture laws used as well as species data for transport estimations (collision diameter and LennardJones potential) are given in a previous work [24] and skipped here for brevity.

We consider aluminum burning in oxygen-containing environments. The reaction mechanism used for gasphase reactions is presented in Tab. 1 (preexponential constant $A$, temperature exponent $b$ and activation energy $E$ ). It is a subset of the more complete mechanism as used in Glorian et al. [24]. It includes 12 gas-phase reactions with 9 species. Although gaseous $\mathrm{Al}_{2} \mathrm{O}_{3}$ is included as an intermediate species, reaction (R12) models a fast condensation into smoke $\mathrm{Al}_{2} \mathrm{O}_{3(l)}$. The mass fraction of gaseous alumina never exceeds $10^{-6}$ in our computations so that most alumina is present under a liquid form. In addition to this gas-phase mechanism, an evaporation reaction is added as $\mathrm{Al}_{(l)}=\mathrm{Al}_{(g)}$ with rate $\dot{\omega}_{\text {vap }}$ given by a Hertz-Knudsen relation with unit sticking coefficient (see Glorian et al. [24] for details).

\subsection{Treatement of alumina smoke}

Liquid alumina $\mathrm{Al}_{2} \mathrm{O}_{3(l)}$ condenses as fine particles $\left(d_{o x} \approx 1 \mu \mathrm{m}\right)$ with small inertia. A Stokes number $S t$ is here defined as the ratio between the inertial time scale of the smoke phase over the flow time scale at the burning aluminum particle scale

$$
S t=\frac{\tau_{\text {inertia }}}{\tau_{\text {flow }}}=\frac{\rho_{\text {ox }} d_{o x}^{2} C_{c}}{18 \mu} \frac{u_{A l}}{d_{A l}}
$$

Table 1

Gas-phase reaction mechanism used (units: cm-mol-s-K)

\begin{tabular}{lllll}
\hline Number & Reaction & $A$ & $b$ & $E / R$ \\
\hline 1 & $\mathrm{Al}+\mathrm{O}+\mathrm{M}=\mathrm{AlO}+\mathrm{M}$ & $3.00 \mathrm{E} 17$ & -1 & 0 \\
2 & $\mathrm{Al}+\mathrm{O}_{2}=\mathrm{AlO}+\mathrm{O}$ & $9.72 \mathrm{E} 13$ & 0 & 80 \\
3 & $\mathrm{AlO}+\mathrm{O}_{2}=\mathrm{AlO}_{2}+\mathrm{O}$ & $4.62 \mathrm{E} 14$ & 0 & 10008 \\
4 & $\mathrm{O}_{2}+\mathrm{M}=\mathrm{O}+\mathrm{O}+\mathrm{M}$ & $1.20 \mathrm{E} 14$ & 0 & 54244 \\
5 & $\mathrm{Al}_{2} \mathrm{O}_{3}=\mathrm{Al}_{2} \mathrm{O}_{2}+\mathrm{O}$ & $3.00 \mathrm{E} 15$ & 0 & 49144 \\
6 & $\mathrm{Al}_{2} \mathrm{O}_{3}=\mathrm{AlO}+\mathrm{AlO}$ & $3.00 \mathrm{E} 15$ & 0 & 63915 \\
7 & $\mathrm{Al}_{2} \mathrm{O}_{2}=\mathrm{AlO}+\mathrm{AlO}$ & $1.00 \mathrm{E} 15$ & 0 & 59336 \\
8 & $\mathrm{Al}_{2} \mathrm{O}_{2}=\mathrm{Al}+\mathrm{AlO}$ & $1.00 \mathrm{E} 15$ & 0 & 74937 \\
9 & $\mathrm{Al}_{2} \mathrm{O}_{2}=\mathrm{Al} l_{2} \mathrm{O}+\mathrm{O}$ & $1.00 \mathrm{E} 15$ & 0 & 52466 \\
10 & $\mathrm{AlO}_{2}=\mathrm{AlO}+\mathrm{O}$ & $1.00 \mathrm{E} 15$ & 0 & 44565 \\
11 & $\mathrm{Al}_{2} \mathrm{O}=\mathrm{AlO}+\mathrm{Al}$ & $1.00 \mathrm{E} 15$ & 0 & 67036 \\
12 & $\mathrm{Al}_{2} \mathrm{O}_{3}=\mathrm{Al} \mathrm{O}_{3(l)}$ & $1.00 \mathrm{E} 15$ & 0 & 0 \\
\hline
\end{tabular}

where $u_{A l}$ is the flow velocity at the aluminium particle surface. For computations presented in the following section $\left(d_{A l}=70 \mu \mathrm{m}\right.$ at $p=1$ atm $)$, we find a maximum value of $S t \approx 0.5$. This suggests that the inertia of smoke particles is quite limited. Thermal equilibrium can similarly be inferred. Smoke particles are then considered as a passive tracer that can be followed using a mass fraction $Y_{o x}$ given by a simple convection equation (without mass diffusion, i.e. $\mathcal{D}_{k}$ is set to zero). Although this mirrors the treatment of a gas-phase species, this is not exactly the case. In fact, we do account for the condensed nature of smoke in thermodynamics or transport for instance. Since smoke volume fraction is always small (below $1 \%$, even if mass fraction is large), it is supposed not to affect transport quantities (viscosity, thermal conductivity and mass diffusion). On the contrary, mixture density is significantly increased due to the high density of this liquid phase. In all the equations presented in the previous section, quantities such as density, velocity, etc. must therefore be seen as average gas/liquid mixture quantities.

Thermophoresis and diffusiophoresis are handled similarly by adding the additional convective fluxes $\rho Y_{o x} \mathbf{v}_{d}$ and $\rho Y_{o x} \mathbf{v}_{t h}$ to the conservation equation on $Y_{o x}$.

\subsection{Numerical procedure}

The global conservative equations Eq. (16) are solved using our in-house code CPS, which is mostly used for aerospace flows $[48,24,9,10]$. This code adopts a finite-volume technique on unstructured mesh. Conservative variables $\mathbf{U}$ are calculated at the center of each computational cell whereas convective and diffusive fluxes are computed at cell edges using an approximate Riemann solver adapted for multi-species flows (Roe-Toumi scheme [49]). Positivity and monotonicity 
of mass fractions $Y_{k}$ are enforced using Larrouturou's method [50]. Computations are second-order accurate in space using a MUSCL approach with Min-Mod flux limiter and second-order accurate in time using an explicit two-step Runge-Kutta time stepping. Typical CFL numbers used are about 0.5 .

An operator splitting is used for solving gas chemical source terms. First, conservative variables $\mathbf{U}^{n}$ are solved in Eq. (16) without source terms $(\mathbf{S}=0)$ to an intermediate state $\mathbf{U}^{*}$ for time step $\Delta t$. Then, the differential equation

$$
\frac{d \mathbf{U}}{d t}=\mathbf{S}
$$

is solved from $\mathbf{U}^{*}$ to the final state $\mathbf{U}^{n+1}$. Since the source term $\mathbf{S}$ is stiff, this differential equation is solved using an implicit Runge-Kutta-Rosenbrock method.

\subsection{Geometry and boundary conditions}

Most computations performed in this work will focus on an aluminum particle with diameter $d_{A l}=70 \mu \mathrm{m}$ because it is an average diameter of particles studied in our past experiments $[34,35,16]$ and also because it is a typical size of aluminum agglomerates released during solid propellant combustion. We consider a quiescent environment without any imposed flow. In that case, the position of the lobe is irrelevant and we consider an axisymmetric configuration just as sketched in Fig. 4. The gas domain is meshed with square elements with grid clustering close to the surface: the smallest grid spacing is about $0.5 \mu \mathrm{m}$ on particle surface (about $d_{A l} / 140$ ). The grid extends up to $25 d_{A l}$ and the particle surface is discretized using approximately 100 elements. This gives a total number of grid points of about 30,000. Grid convergence was checked by considering a coarser mesh $(11,000$ elements, smallest grid spacing $1 \mu \mathrm{m})$ and the quantity of interest (i.e., thermophoretic flux) was off by only $3 \%$. Geometry is fixed in time (i.e., particle regression is not explicitly tracked) due to much longer burnback time scales compared to flow time scales. Figure 6 presents an example of the grid used for the case $d_{A l}=70 \mu \mathrm{m}$ and $f_{o x}=0.3$.

Remote far-field boundary conditions are prescribed pressure $p^{\infty}=1$ atm, zero gas velocity, temperature $T^{\infty}=300 \mathrm{~K}$ and $\mathrm{O}_{2} /$ Ar mixture (0.2/0.8 molar).

The aluminum particle with its lobe therefore includes two boundary conditions: one evaporation condition for aluminum and a specific no-slip conditions for the oxide lobe-which is described hereafter. At

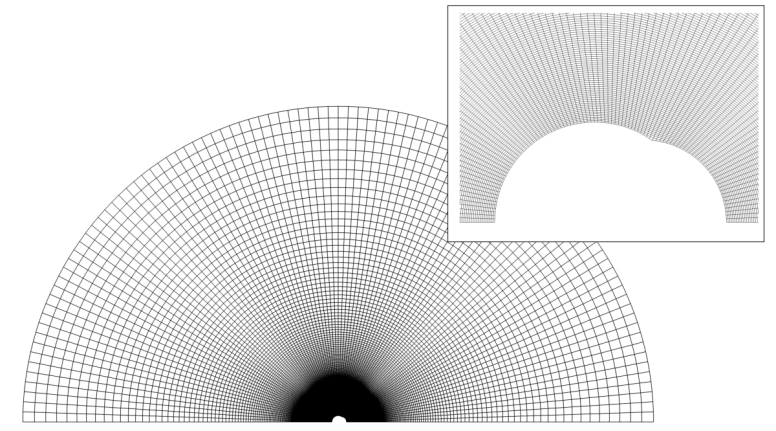

Figure 6: Example of grid used $\left(d_{A l}=70 \mu \mathrm{m}, f_{o x}=0.3\right)$. Inset: close-up view on the particle. The oxide lobe is on the right side.

the aluminum surface, flux-matching conditions are required to obtain surface species $Y_{k}^{s}$ and surface temperature $T^{s}$. Species mass fractions at surface $Y_{k}^{s}$ are obtained by a balance between convective, diffusive fluxes and surface production rates $\dot{\omega}_{k}^{s}$

$$
\dot{\omega}_{\text {vap }} Y_{k}^{s}-\left.\rho \mathcal{D}_{k} \frac{d Y_{k}}{d n}\right|_{s}=-\dot{\omega}_{k}^{s}
$$

Because there are no surface reactions considered here apart from aluminum vaporization, we have $\dot{\omega}_{k}^{s}=\dot{\omega}_{\text {vap }}$ for $\mathrm{Al}_{(g)}$ and 0 otherwise. Surface temperature arises from the conservation of energy at the aluminum surface

$$
-\left.\lambda \frac{d T}{d n}\right|_{s}=\dot{\omega}_{\text {vap }} h_{A l_{(g)}}
$$

where $h$ is the enthalpy.

\subsection{Deposition boundary condition for the lobe}

For the oxide lobe, a specific boundary condition is developed. It is basically a no-slip condition with a fixed temperature, imposed to the temperature of the aluminum due to isothermal assumption. In addition, the alumina deposition normal mass flux $j_{d e p}\left(\mathrm{~kg} \cdot \mathrm{m}^{-2} \cdot \mathrm{s}^{-1}\right)$ on the lobe is computed. Four mechanisms are accounted for: convection (subscript "conv"), Brownian ("br"), thermophoresis ("th") and diffusiophoresis ("d"):

$$
j_{\text {dep }}=j_{\text {conv }}+j_{b r}+j_{t h}+j_{d}
$$

In the following expressions, all quantities are estimated in the neighboring cell adjacent to the surface of the lobe. The thermophoresis and diffusiophoresis mass flux can be computed by

$$
\begin{aligned}
j_{t h} & =-\rho Y_{o x} \mathbf{v}_{t h} \cdot \mathbf{n} \\
j_{d} & =-\rho Y_{o x} \mathbf{v}_{d} \cdot \mathbf{n}
\end{aligned}
$$


where $\mathbf{n}$ is a local normal vector on the lobe pointing outwards. The Brownian flux reads

$$
j_{b r}=-\rho \mathcal{D}_{b r} \frac{d Y_{o x}}{d n}
$$

where $d Y_{o x} / d n=\nabla Y_{o x} \cdot \mathbf{n}$ is the normal gradient of $Y_{o x}$ at the surface while the Brownian diffusion coefficient is given as

$$
\mathcal{D}_{b r}=C_{c} \frac{k_{b} T}{3 \pi \mu d_{o x}}
$$

with $k_{b}$ the Boltzmann constant. Finally, the deposition mass flux arising from the convection of the mean flow is expressed as

$$
j_{\text {conv }}=-\rho Y_{o x} \eta_{\text {coll }} \mathbf{v} \cdot \mathbf{n}
$$

where $\eta_{\text {coll }}$ is the collision efficiency, i.e. the probability that fine smoke particles effectively collide with the lobe (rather than circumventing it). This reflects the inertia of smoke relative to the flow and is related to a Stokes number $S t$ defined in a similar way that as in Eq. (26), instead that lobe diameter is used instead of aluminum particle diameter. We take the expression from Salita [51], which was specifically devised for alumina smoke as

$$
\eta_{\text {coll }}=\frac{S t^{1.26}}{1.26+S t^{1.26}}
$$

The coalescence efficiency is assumed equal to 1 , i.e. smoke particles effectively merge and feed the oxide lobe upon collision, which can be easily justified by the small impact Weber numbers. When mass fluxes are computed to be negative (i.e., smoke is carried out away from the surface), they are set to zero. The total deposited mass rate $\dot{m}_{d e p}\left(\mathrm{~kg} . \mathrm{s}^{-1}\right)$ is computed by integrating the mass flux over all computational cells discretizing the lobe:

$$
\dot{m}_{d e p}=\int_{S_{o x}} j_{d e p} \cdot d S
$$

The average deposition flux $\left\langle j_{d e p}\right\rangle$ is estimated as $\left\langle j_{d e p}\right\rangle=\dot{m}_{d e p} / S_{o x}$ where $S_{o x}$ is the total surface of the oxide lobe. In each computational cell on the particle surface, the computed smoke mass flux $j_{d e p} \cdot \mathbf{n}$ is applied as a boundary condition for the numerical flux $\rho Y_{o x} \mathbf{v}$ so as to effectively mimic an actual deposition on the particle and remove smoke from the flow.

\section{Results}

\subsection{Reference particle - No lobe}

We start with a spherical particle without any lobe (i.e., $f_{o x}=0$ ). Although hypothetical (burning aluminum particles have generally a significant lobe, even at the early stages of combustion), this configuration is instructive as it illustrates how thermophoresis is a powerful mechanism for smoke deposition, even in the absence of lobe.

As already specified in Sec. 4, we consider simulations on a spherical particle with diameter $d_{o x}=70 \mu \mathrm{m}$ burning in a $\mathrm{Ar} / \mathrm{O}_{2}(80 / 20)$ mixture at $p=1$ atm. In this particular case, we compute the different deposition mass rates on the particle rather than on the lobe. A simulation run without any thermo- and diffusiophoresis leads to the absence of convective deposition: $\left\langle j_{\text {conv }}\right\rangle=0$ due to the outward Stefan flow and a vanishing Brownian contribution $\left\langle j_{b r}\right\rangle \sim 5.10^{-6} \mathrm{~kg} \cdot \mathrm{m}^{-2} . \mathrm{s}^{-1}$ because of the very low alumina content on particle surface (recalling that Fickian diffusion of smoke is dismissed). However, activating thermophoretic motion yields $\left\langle j_{t h}\right\rangle=0.39 \mathrm{~kg} . \mathrm{m}^{-2} . \mathrm{s}^{-1}$, which is a significant fraction of the total evaporation mass rate of aluminum due to combustion $\left\langle j_{A l}\right\rangle=2.77 \mathrm{~kg} \cdot \mathrm{m}^{-2} \cdot \mathrm{s}^{-1}$. It is more pertinent to estimate the fraction of alumina coming back to the aluminum particle with respect to the total alumina produced. Noting $v_{s t}$ the mass stoichiometric coefficient of aluminum to alumina $\left(v_{s t}=1.89\right)$, the total production rate of alumina is then $v_{s t}\left\langle j_{A l}\right\rangle$ and the fraction $f_{d e p}$ of deposited alumina to the total alumina production reads $f_{d e p}=\left\langle j_{d e p}\right\rangle / v_{s t}\left\langle j_{A l}\right\rangle$. In this case, we find $f_{\text {dep }}=0.075$ meaning that $7.5 \%$ of the total alumina produced moves back to the particle surface due to thermophoresis. Note that if we assume $f_{\text {dep }}$ constant throughout the combustion, the final oxide residue size ratio $\beta$ (as discussed in the introduction) would simply be $\beta=\left(v_{s t} f_{d e p} \rho_{A l} / \rho_{o x}\right)^{1 / 3}=0.44$. This is already a significant value, showing that thermophoresis by itself is active in contributing to oxide lobe and residue formation. This effect was probably underrated in previous works because many authors surmised that the Stefan flow due to aluminum evaporation would be effective in hindering any phoretic motions. In fact, thermophoretic velocity in this particular case is $30 \mathrm{~m} / \mathrm{s}$-well above the outward Stefan flow $(12 \mathrm{~m} / \mathrm{s})$ - because of high thermal gradients. The particle surface temperature is computed to be $2570 \mathrm{~K}$ in those conditions while the flame temperature is over $3800 \mathrm{~K}$.

If we both include thermophoresis and diffusiophoresis, the total deposited mass rate $\left\langle j_{d e p}\right\rangle$ decreases from $0.39 \mathrm{~kg} \cdot \mathrm{m}^{-2} \cdot \mathrm{s}^{-1}$ (thermophoresis only) to 0.36 $\mathrm{kg} \cdot \mathrm{m}^{-2} \cdot \mathrm{s}^{-1}$ (with thermo- and diffusiophoresis), leading to a slight decrease of $f_{\text {dep }}=0.07$ and $\beta=0.42$. This adverse effect of diffusiophoresis can be explained by species diffusive fluxes on surface. The major diffusion fluxes come from gaseous $\mathrm{Al}$ (outwards) and $\mathrm{Ar}$ 
(inwards) but since $\mathrm{Al}$ flux is higher, this results in an overall effect of slightly moving smoke away from the surface.

Because smoke size is not perfectly known, we have investigated smaller smoke diameters $d_{o x}$. Since alumina smoke is expected to form by clustering of alumina molecules, very fine particles are likely to be present. We have actually found little effect of smoke size on thermophoresis (diffusiophoresis is intrinsically size-independent). Smoke size changes the thermophoretic velocity through the Knudsen number in the thermophoretic constant $K_{t h}$ in Eq. (2). However, $K_{t h}$ rapidly levels off at an asymptotic value $\left(K_{t h} \approx 0.55\right)$ for high enough $K n$ (typically about 1 ) which is basically reached for $d_{o x}=1 \mu \mathrm{m}$. Smaller sizes then do not lead to any further changes.

We finally explore the effect of aluminum particle size $d_{A l}$ by running simulations with only thermophoretic and thermophoretic/diffusiophoretic models for aluminum particle size in the range 20 400 $\mu \mathrm{m}$. Particles smaller than $20 \mu \mathrm{m}$ are not considered since surface reactions could play significantly [24] but were not included here. Simulation results are presented in Fig. 7 where the deposited-to-produced fraction $f_{\text {dep }}$ is plotted against aluminum particle size $d_{A l}$. This fraction is noted to decrease with aluminum particle size. This is expected because combustion in this size range is mostly driven by a diffusion flame so that flame temperature $T_{f}$ and surface temperature $T_{s}$ are relatively size-independent. Therefore the temperature gradient $\nabla T \propto\left(T_{f}-T_{s}\right) / d_{A l}$ decreases as $d_{A l}$ goes up. For small particles, diffusion becomes more intense which tends to smooth out thermal gradients. This explains why the increase in $f_{\text {dep }}$ seems less marked for smallest particles. Diffusiophoresis remains a second-order effect and here always tends to reduce $f_{d e p}$, i.e. acts against thermophoresis.

\subsection{Effect of lobe on the flow}

It has been shown previously that thermophoresis is operative in moving smoke back to particle surface against the Stefan flow. Its effect is likely to be even stronger in the lobe region where no outward flow released from aluminum evaporation may hinder smoke motion. Since the composite aluminum/alumina droplet is supposed isothermal, the lobe has a low temperature (here, $2570 \mathrm{~K}$ ) compared to surrounding gas and thermophoresis remains significant. Also, the presence of the lobe may locally modify the flow field, notably temperature and smoke fraction. This is the objective of this section where we focus on a constant $d_{A l}=70 \mu \mathrm{m}$ aluminum particle with different lobe size, expressed in

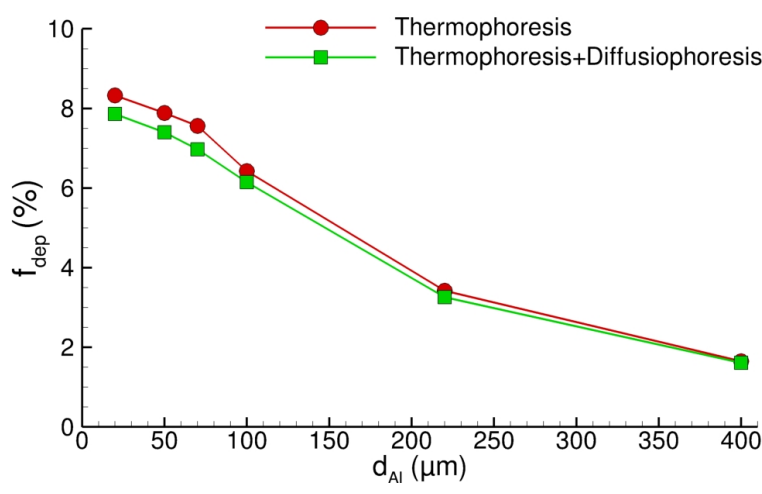

Figure 7: Fraction $f_{\text {dep }}$ of produced alumina deposited on a burning aluminum particle of diameter $d_{A l}$ including thermophoresis only and thermophoresis+diffusiophoresis.

terms of alumina mass fraction $f_{o x}=0.1,0.3,0.5$, and 0.7 as already depicted in Sec. 3.2 (see Fig. 5).

Simulations are run with and without phoretic (i.e., both thermo- and diffusiophoresis) motions. Figure 8 presents a map of oxide smoke mass fraction $Y_{o x}$ obtained at steady state without phoretic motion (a) and with phoretic motion and deposition model (b) for case $f_{\text {ox }}=0.3$. In both cases, the spherical symmetry is clearly broken due to the oxide lobe. There is still an inward motion of fresh gas in the vicinity of the lobe but no Stefan flow. Consequently, this blows the flame back to the lobe, bringing large amount of alumina there. Activating the deposition model with phoretic motion (b) leads to significant changes in the oxide distribution. Thermophoresis tends to smooth out the fraction of smoke away from the flame, where the temperature is maximum. Because of significant deposition rates (on the particle, but especially on the lobe as it will be seen in the next section), the oxide fraction is greatly reduced in the lobe region. The computed maximum fraction of smoke goes down from 0.90 (a) to 0.81 (b) primarily because of smoke removed by deposition. There is no difference noticed whether diffusiophoresis was activated or not, which is consistent with the weak role of diffusiophoresis.

To gain further insight, we focus on this latter case (b), with thermophoresis activated, and plot the thermophoretic mass flux $j_{t h}=\rho Y_{o x}\left|\mathbf{v}_{t h}\right|$ in Fig. 9. The white dotted line is the separating streamline, delineating inward and outward thermophoretic motion (thereby, the locus of maximum temperature). The arrows indicate the direction of motion, which is basically radial, except at the tip of the lobe, where smoke turns inwards. This is reminiscent of the experimental observations presented in Fig. 1 with an intense radial motion to the lobe to- 


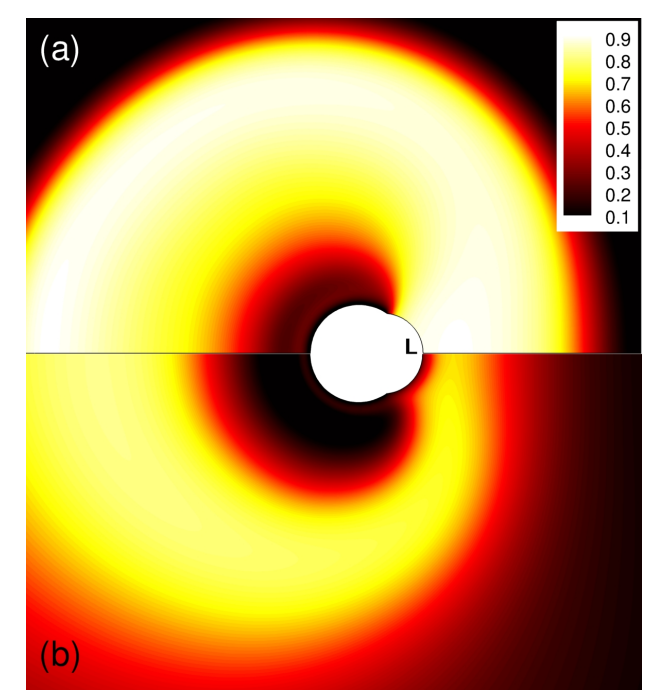

Figure 8: Oxide smoke mass fraction $Y_{o x}$ without (a) and with phoretic motion (b) $\left(f_{o x}=0.3\right)$. The lobe is indicated by " $\mathrm{L}$ "

gether with a transverse motion from the flame. Mass flux values are about $0.4 \mathrm{~kg} \cdot \mathrm{m}^{-2} \cdot \mathrm{s}^{-1}$ on the burning surface (as already computed in Sec. 5.1) but can be as high as $1 \mathrm{~kg} \cdot \mathrm{m}^{-2} \cdot \mathrm{s}^{-1}$ on the lobe, especially at the junction with the particle where mass fluxes are the largest.

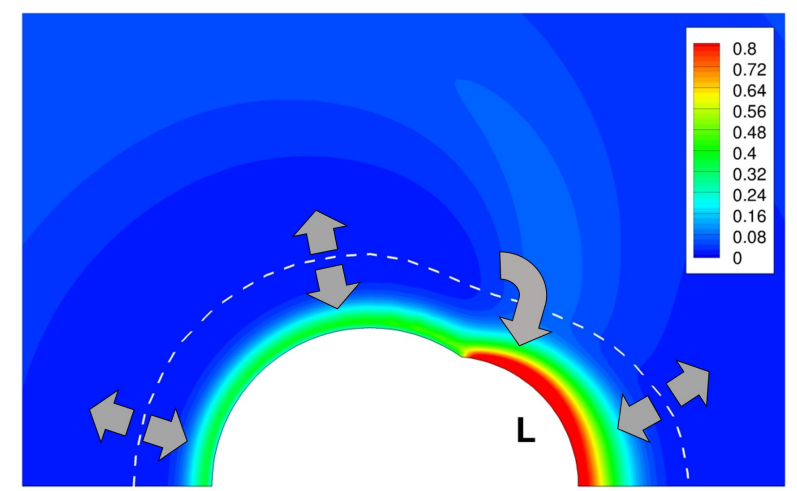

Figure 9: Thermophoretic mass flux $\rho Y_{o x}\left|\mathbf{v}_{t h}\right|\left(f_{o x}=0.3\right)$. The dotted white line is the separating stream line and arrows indicate the smoke motion. The lobe is indicated by " $\mathrm{L}$ ".

In the Fig. 10, a map of oxide smoke mass fraction $Y_{o x}$ is presented for the four lobe sizes investigated. The burning aluminum particle is $d_{A l}=70 \mu \mathrm{m}$ for all cases. As expected, the breaking of fore-aft symmetry is more pronounced for larger lobes, especially the case $f_{o x}=0.7$. The maximum value of $Y_{o x}$ decreases with $f_{o x}$, from $0.83\left(f_{o x}=0.1\right)$ down to $0.66\left(f_{o x}=0.7\right)$, because more smoke is captured and removed from the flow. Interestingly, the aluminum evaporation rate is found to remain virtually unchanged, with only a $2 \%$ variation. This means that aluminum combustion is not altered by the presence of the lobe, at least within our assumptions (in particular, no heat transfer between aluminum and oxide lobe).

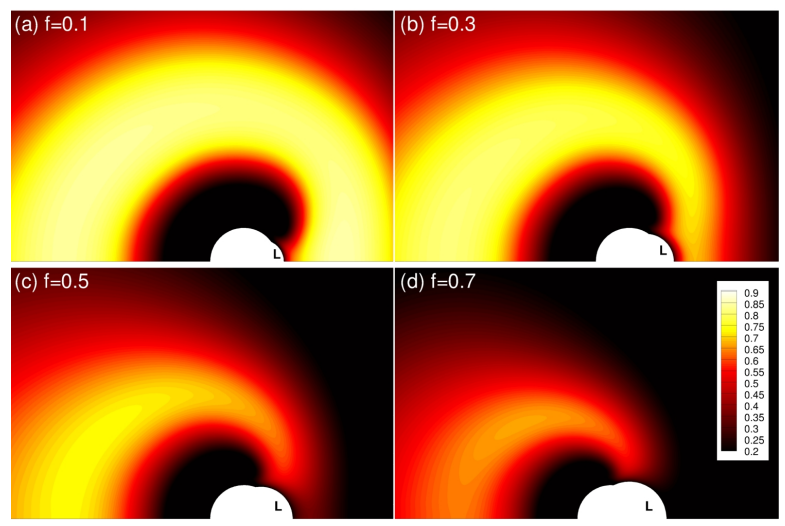

Figure 10: Oxide smoke mass fraction $Y_{o x}$ for a $70 \mu \mathrm{m}$ aluminum particle with different lobe size (alumina mass fraction $f_{o x}=0.1$ (a), 0.3 (b), 0.5 (c), and 0.7 (d)). The lobe is indicated by "L". Identical contour levels are used.

\subsection{Effect of lobe on deposition rates}

We here report on the deposition rates obtained from our simulations for the four mass alumina fractions investigated. Table 2 presents the average mass flux (broken into the different mechanisms) deposited on the lobe depending on the lobe extent. The first surprising result is that deposition by convection is negligible. This is basically due to the minimal gas velocity in the vicinity of the lobe (below $0.1 \mathrm{~m} / \mathrm{s}$ ) together with small collision efficiency $\eta_{\text {coll }}$. Because of negligible inertia, particles then flow around the lobe without colliding. Even if we artificially imposed $\eta_{\text {coll }}=1$, the flux would reach a mere $0.01 \mathrm{~kg} \cdot \mathrm{m}^{-2} \cdot \mathrm{s}^{-1}$. It is however important to underline that we have supposed a quiescent particle. During actual burning, the particle may switfly and erratically move and spin, which offers opportunities for sweeping nearby smoke by the lobe relative motion. In addition, a relative gas flow loaded with smoke (as in an actual solid rocket chamber) could also involve deposition by convection on the windward lobe. All of those mechanisms were discarded here.

Likewise, contribution from Brownian motion is found negligible. Actually, the most effective deposition mechanism is thermophoresis and we here again confirm a limited contribution from diffusiophoresis. Note however that this time diffusiophoresis acts synergistically with thermophoresis due to inward motion 
of oxygenated species (mostly, $\mathrm{O}$ and $\mathrm{O}_{2}$ ). The thermophoretic mass flux remains high for all lobe sizes and is typically three times more intense on the lobe than on the burning particle. We recall that presented values are the surface-averaged mass flux and because the oxide lobe surface $S_{o x}$ grows with $f_{o x}$, the overall mass rate of deposited oxide increases with $f_{o x}$.

Those results, together with the contour maps from Fig. 10, can help propose a first physical picture: because the lobe does not outgas, the flame (thereby, high fractions of smoke and high temperatures) is swept back to the lobe region. Resulting higher temperature gradients and large amount of smoke hence boost an intense thermophoretic motion of nearby smoke to the lobe. The mechanism has therefore both aerodynamic and phoretic origins.

Table 2

Average oxide mass rates deposited on the lobe $\left(\mathrm{kg} \cdot \mathrm{m}^{-2} \cdot \mathrm{s}^{-1}\right)$

\begin{tabular}{llllll}
\hline$f_{\text {ox }}$ & $\left\langle j_{\text {conv }}\right\rangle$ & $\left\langle j_{\text {br }}\right\rangle$ & $\left\langle j_{\text {th }}\right\rangle$ & $\left\langle j_{d}\right\rangle$ & total \\
\hline 0.1 & $\sim 10^{-4}$ & $\sim 10^{-5}$ & 1.08 & 0.10 & 1.18 \\
0.3 & $\sim 10^{-4}$ & $\sim 10^{-5}$ & 0.99 & 0.06 & 1.05 \\
0.5 & $\sim 10^{-4}$ & $\sim 10^{-5}$ & 0.73 & 0.04 & 0.77 \\
0.7 & $\sim 10^{-4}$ & $\sim 10^{-5}$ & 0.81 & 0.03 & 0.84 \\
\hline
\end{tabular}

At this point, we have computed the mass flux of oxide deposited on the lobe $\left\langle j_{d e p}^{o x}\right\rangle$ (Tab. 2) but we also know (from Sec. 5.1) the oxide mass flux coming directly on the burning aluminum particle (here, noted $\left.\left\langle j_{\text {dep }}^{\text {part }}\right\rangle\right)$. It is then possible to compute the overall oxide deposition fraction $f_{\text {dep }}$ by summing up the contribution from the aluminum particle and from the lobe as

$$
f_{d e p}=\frac{\left\langle j_{d e p}^{\text {part }}\right\rangle S_{A l}+\left\langle j_{d e p}^{o x}\right\rangle S_{o x}}{v_{s t}\left\langle j_{A l}\right\rangle S_{A l}}
$$

where $S_{A l}$ is the surface of the aluminum particle and $S_{o x}$ the surface of the lobe, with expressions given by Eqs. (14)-(15). The obtained deposition fraction is presented in Fig. 11 as a function of $f_{o x}$. Because the case $f_{o x}=0.7$ showed a rapid increase in $f_{d e p}$, we have computed an additional geometry $f_{o x}=0.9$ to confirm this trend. We do observe an increase in the deposition fraction with lobe size. It is moderate for small lobes but much more marked for high $f_{o x}$, typically higher than 0.6. This might be connected with the position of the smoke trail, which is now entirely above the lobe (as in Fig. 10(d)), thereby maximizing thermophoresis mass rate. More computations, with different $f_{o x}$ would however be required to propose a conclusive explanation. Overall, the deposited ratio is in the range 10 20\% during the early stages of combustion before increasing to larger values at the end of burning.

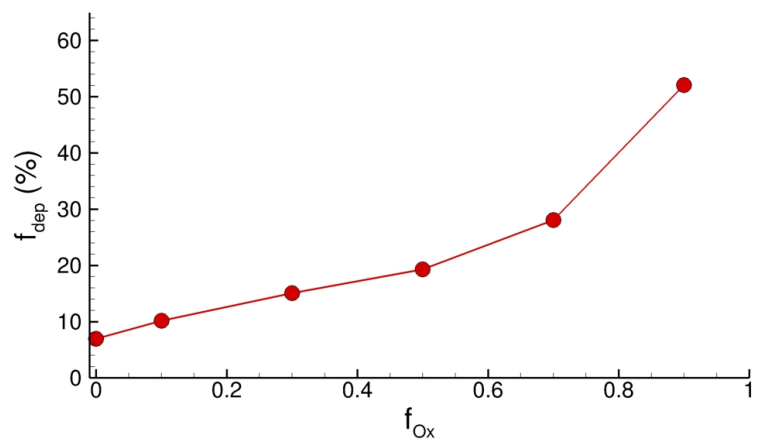

Figure 11: Fraction $f_{\text {dep }}$ of produced alumina deposited on the lobe and particle as a function of lobe size $f_{o x}$.

\subsection{Prediction of aluminum oxide residue}

Results presented heretofore are sufficient to propose a preliminary model able to provide quantitative predictions of lobe growth, hence the size of final oxide residues. Let us start with an aluminium particle of diameter $d_{A l}^{0}$ containing an initial oxide fraction $f_{o x}^{0}$. The evolution of aluminium $V_{A l}$ and oxide $V_{O x}$ volumes are then given as

$$
\begin{aligned}
\frac{d V_{A l}}{d t} & =-\frac{\left\langle j_{A l}\right\rangle S_{A l}}{\rho_{A l}} \\
\frac{d V_{o x}}{d t} & =+\frac{v_{s t} f_{d e p}\left\langle j_{A l}\right\rangle S_{A l}}{\rho_{o x}}
\end{aligned}
$$

Once volumes are known-and so is $f_{o x}$-all geometrical data (in particular, particle and lobe surfaces $S_{A l}$ and $S_{o x}$ ) are deduced from the geometrical reconstruction procedure as detailed in Sec. 3.2. Hence, to close the model, we need to prescribe constitutive relations for $\left\langle j_{A l}\right\rangle$ as well as for $f_{d e p}$. When a $d^{2}$ law is assumed, the scaling $\left\langle j_{A l}\right\rangle \propto 1 / d_{A l}$ is expected. This is confirmed by our simulations on different aluminum particle sizes, except for the smallest case $20 \mu \mathrm{m}$, where a transition to a kinetically-limited regime is noted. Therefore, the relation $\left\langle j_{A l}\right\rangle=2.77 \times\left(70.10^{-6} / d_{A l}\right)$ is chosen from simulations.

Concerning $f_{d e p}$, we have seen that it depends much on $f_{o x}$ (at constant $d_{A l}$, see Fig. 11) but is also related on $d_{A l}$ as suggested by Fig. 7. A thorough characterization of the relation $f_{d e p}=F\left(d_{A l}, f_{o x}\right)$ would require a significant number of computations not done in the frame of this study. As a first guess, we assume a multiplicative effect $f_{d e p}=g\left(d_{A l}\right) \cdot h\left(f_{o x}\right)$ with $h$ is the law directly taken from results in Fig. 11. We further correct from size effects through function $g$ obtained-as a 
first simplification - from a linear regression on results presented in Fig. 7. We are aware that this model is a crude approximation that needs to be refined by further simulations but this may be sufficient for first estimations. In particular, the assumption of a $d^{2}$ law as well as considering the direct simulation results obtained for large particles may be questionable for the smallest diameters, where a diffusional regime may no longer hold. Solving Eqs. (38)-(39) with prescribed functions $\left\langle j_{A l}\right\rangle$ and $f_{\text {dep }}$ finally yields the time evolution of the burning particle and lobe growth.

Figure 12 presents the evolution of the aluminum and oxide particle size with time for the combustion of a $d_{A l}^{0}=70 \mu \mathrm{m}$ particle with initial oxide fraction $f_{o x}^{0}=0$ as computed from the previous model. Since particles are not spheres, we here plot a volume-equivalent diameter $d=(6 V / \pi)^{1 / 3}$ as a more relevant parameter. (Indeed, small oxide lobes may have a vanishingly small volume but finite $d_{o x}$.) For this particular case, the predicted burning time is $14.4 \mathrm{~ms}$, which is longer than a particle without lobe (dotted lines) that would give $11.3 \mathrm{~ms}$. Oxide lobe slows combustion down due to the reduced available surface for aluminum evaporation. The predicted size of oxide residue $d_{\text {res }}$ - when aluminum has fully burnt out - is about $39 \mu \mathrm{m}$, which gives a size ratio $\beta=d_{\text {res }} / d_{A l}^{0}=0.55$. This is an encouraging result since it is in line with most experimental data, between 0.5 and 0.8 , as discussed in the introduction. This definitely supports that smoke deposition by thermophoresis is a dominant mechanism for lobe growth.

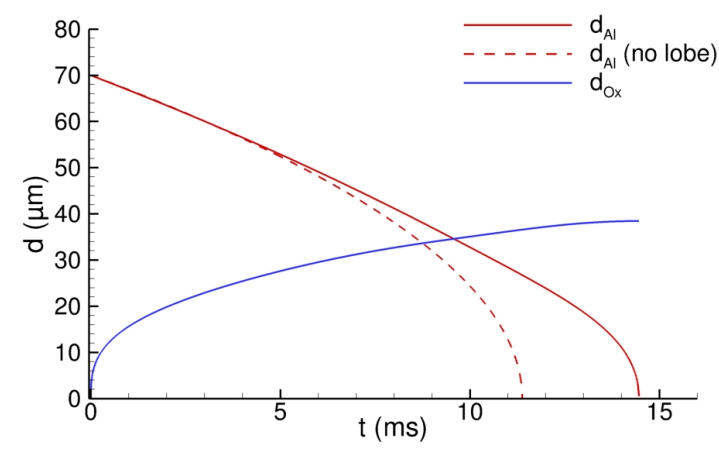

Figure 12: Predicted equivalent particle diameter for aluminum and oxide with time $\left(d_{A l}^{0}=70 \mu \mathrm{m}, f_{o x}^{0}=0\right)$. Dotted line: aluminum without lobe.

The effect of aluminum particle size has been investigated using this simple model and we find that size ratio $\beta$ decreases with initial aluminum size $d_{A l}^{0}$. For small particles $\left(d_{A l}^{0}<50 \mu \mathrm{m}\right)$, the size ratio $\beta$ seems to reach an asymptotic value $\beta=0.57$. The effect is mostly appreciable for larger particles with $\beta=0.49$ for $d_{A l}^{0}=200 \mu \mathrm{m}$ and $\beta=0.40$ for $d_{A l}^{0}=400 \mu \mathrm{m}$.

Actually, the most significant effect is related to the initial presence of a lobe, i.e. the value of $f_{o x}^{0}$. The usual assumption of an aluminum particle passivated by an oxide layer of size $\sim 5 \mathrm{~nm}$ would give virtually no initial lobe with an estimated $f_{o x}^{0} \approx 10^{-4}$. This contrasts with our experiments $[35,16]$ where-depending on burning conditions - a significant lobe exists right after melting, before burning inception. Babuk et al. [19] proposed that oxide mass fraction can go up to to $30 \sim 40$ $\%$ by the beginning of gas-phase burning. This significant oxide production is believed to result from an initial intense heterogeneous stage. Table 3 presents the predicted burning time $t_{b}$ and oxide residue size ratio $\beta$ with respect to the initial oxide mass fraction $f_{o x}^{0}$. We also define a modified size ratio $\tilde{\beta}$ which is based on the final oxide residue size subtracted from the initial oxide volume $V_{o x}^{0}$, i.e. $d_{\text {res }}=\left(6\left(V_{o x}-V_{o x}^{0}\right) / \pi\right)^{1 / 3}$. This hypothetical quantity is thus a measure of the effectively deposited oxide and allows to gauge the effect of the deposition itself, unbiased by the initial amount of oxide prior to burning. The results point out a crucial importance of initial oxide fraction, which tends to provide slower combustion and much larger oxide residues. Even for a small $f_{o x}^{0}=0.1-$ a value visually compatible with experimental observations - the size ratio jumps from 0.55 to 0.7 . Of course, this is partly in connection with more oxide present initially on the particle but the fact that $\tilde{\beta}$ has also increased (0.61) indicates that the deposition mechanism is enhanced by an initial lobe which promotes larger deposition rates at the very beginning of combustion. Nonetheless, experimental measurements (via laser granulometry or direct visualisation) generally consider the initial diameter of a particle, irrespective of the initial quantity of oxide. This means that, from an experimental perspective, $\beta$ is measured, not $\tilde{\beta}$. It is therefore interesting to note that predicted $\beta$ lies in the range $0.5 \sim 0.8$, exactly as in experiments. This can suggest that the initial extent of the lobe, prior to combustion, could help explain experimental variations. The model finds that $\beta$ can sometimes exceed 1, i.e. residues larger than initial particles, which was already noticed by Dreizin [5]. For a $70 \mu \mathrm{m}$ particle, this is predicted to occur for $f_{o x}^{0}>0.4$. As a summary, the initial lobe plays a major role and this is quite consistent with our experimental observations discussed in Sec. 2.

Let us end with a final remark on the burning time $t_{b}$, which can be largely altered by the lobe. A $70 \mu \mathrm{m}$ aluminum particle is predicted to burn in $11.3 \mathrm{~ms}$ without lobe while slowing down to $16.5 \mathrm{~ms}$ with a lobe 
Table 3

Final oxide residue size ratio $\beta=d_{\text {res }} / d_{A l}^{0}$ against initial oxide content $f_{o x}^{0}$ for a $d_{A l}^{0}=70 \mu \mathrm{m}$ particle at $p=1$ bar. Size ratio $\tilde{\beta}$ is based on the solely deposited oxide, irrespective of the initial oxide.

\begin{tabular}{llll}
\hline$f_{o x}^{0}$ & $t_{b}(\mathrm{~ms})$ & $\beta$ & $\tilde{\beta}$ \\
\hline 0.00 & 14.4 & 0.55 & 0.55 \\
0.05 & 15.6 & 0.64 & 0.59 \\
0.10 & 16.5 & 0.70 & 0.61 \\
0.15 & 17.3 & 0.75 & 0.63 \\
0.20 & 18.0 & 0.81 & 0.65
\end{tabular}

with initial fraction $f_{o x}^{0}=0.1$ - which is almost a $50 \%$ increase. However, we have noted that the combustion still roughly follows a $d^{2}$ law. Fitting burning times in a no-lobe configuration yields a power law with the expected exponent $n=2.00$. However, including the lobe only slightly reduces the size exponent, down to 1.94 . Although the presence of the lobe reduces the available burning surface, its effect is mostly prominent at the end of burning, which represents a small portion of time. Large deviations from a $d^{2}$ law are hence not apparently connected to a purely geometrical effect. This might rather arise from a transition in the combustion regime for small diameters. Direct imaging of particle comb(ustion showed that a $d^{2}$ law was kept during the early stages of combustion (typically until $\approx 20 \mu \mathrm{m}$ ), irrespective of the lobe [35].

\section{Conclusions}

This work reports on direct numerical simulations of a burning aluminum droplet with a detailed geometrical representation of its oxide lobe. A peculiarity of this work is to include phoretic motions of small oxide smoke created during aluminum combustion - basically thermophoresis and diffusiophoresis-without resorting to Fick's law, which is not adequate for smoke. A major outcome is that thermophoresis is predominant in driving smoke back to particle surface, thereby feeding the oxide lobe. The mechanism attested by computations is that the lobe distorts the flame, bringing hot and oxide-rich regions close to the lobe, which favors intense thermophoretic motions towards it. For large lobes, up to half of the total oxide produced can move back to the lobe. Simulation results have been processed to propose a simple zero-dimensional model, which estimates burning times and final oxide residue sizes. Final residue diameters are typically 50 80\% of the initial particle size, which is in good agreement with the scarce experimental data. The size of the initial lobe-presumably produced by a heterogeneous stage prior to gas-phase burning-is found to have an important role. This suggests that chemical surface production of oxide, at the very early stages of combustion, could participate in the overall mechanism as well. It is worth noting that the main simulation results, i.e the role of initial lobe and the intense smoke motion towards the lobe, have been confirmed by direct observations on a single burning aluminum particle. To our knowledge, those results are the first quantitative and physically-supported predictions of final oxide residues. Although they strongly support a prominent thermophoretic-based process, other aspects such as heterogeneous combustion or smoke captured by sweeping due to a jetting/spinning burning particle, have not been considered here and could contribute as well. In addition, rocket environments contain a large amount of oxide, which could contribute to enhance the rate of deposition computed in this work.

Future works need to assess this mechanism for wider burning conditions, including particle size but also pressure for instance. Thermophoresis is expected to be less intense for higher pressures due to reduced Knudsen numbers. Likewise, the nature of oxidizers is worth studying as a possible way to understand experimental differences in lobe size between $\mathrm{N}_{2}$ and Ar environments for instance. Since the initial lobe is believed to be partly produced by pre-burning surface chemical production, the ignition phase should also be studied in more detail, e.g., the effect of ignition time or ignition intensity.

\section{Acknowledgments}

The work was funded by the French Defense Procurement Agency (DGA).

\section{References}

[1] L. Soler, J. Macanás, M. Munoz, J. Casado, J. Power Sources 169 (2007) 144-149.

[2] J. Bergthorson, S. Goroshin, M. Soo, P. Julien, J. Palecka, D. Frost, D. Jarvis, Appl. Energ. 160 (2015) 368-382.

[3] R. Lomba, P. Laboureur, C. Dumand, C. Chauveau, F. Halter, Proc. Combust. Inst. 37 (2019) 3143-3150.

[4] M. Beckstead, RTO/VKI Special Course on Internal Aerodynamics in Solid Rocket Propulsion RTO-EN-023 (2004).

[5] E. L. Dreizin, Combust. Flame 105 (1996) 541-556.

[6] M. Beckstead, Combust. Explo. Shock Waves 41 (2005) 533546.

[7] M. King, Proc. Combust. Inst. 32 (2009) 2107-2114.

[8] M. Salita, 21st JANNAF Exhaust Plume Technology Meeting 1 (1994) 1-17.

[9] S. Gallier, F. Godfroy, J. Prop. Power 25 (2009) 509-521. 
[10] S. Gallier, B. Briquet, M. Yiao, J. Prop. Power 35 (2019) 159172.

[11] A. Genot, S. Gallier, T. Schuller, submitted to J. Prop. Power (2020).

[12] S. Turns, S. Wong, E. Ryba, Combust. Sci. Technol. 54 (1987) 299-318.

[13] O. Glotov, V. Zhukov, Combust. Explo. Shock Waves 44 (2008) 671-680.

[14] A. Zenin, G. Kusnezov, V. Kolesnikov, AIAA Paper 2000-849 (2000).

[15] S. Gallier, J.-G. Kratz, N. Quaglia, G. Fouin, Prog. Propulsion Phys. 8 (2016) 197-212.

[16] A. Braconnier, C. Chauveau, F. Halter, S. Gallier, Exp. Therm. Fluid Sci. (2020) 110110

[17] E. L. Dreizin, Combust. flame 117 (1999) 841-850.

[18] A. Zenin, G. Kusnezov, V. Kolesnikov, AIAA Paper 1999-696 (1999).

[19] V. Babuk, V. Vasilyev, M. Belogub, O. Romanov, 4th International Symposium On special topics in Chemical Propulsion, Stockholm (1996).

[20] V. Babuk, V. Vasilyev, J. Prop. Power 18 (2002) 814-823.

[21] V. E. Zarko, O. G. Glotov, Sci. Technol. Energ. Mat. 74 (2013) 139-143.

[22] J. S. Sabnis, J. Prop. Power 19 (2003) 48-55.

[23] J. Glorian, L. Catoire, S. Gallier, N. Cesco, Proc. Combust. Inst. 35 (2015) 2439-2446.

[24] J. Glorian, S. Gallier, L. Catoire, Combust. Flame 168 (2016) 378-392.

[25] M. Beckstead, Y. Liang, K. Pudduppakkam, Combust. Explo. Shock Waves 41 (2005) 622-638.

[26] E. Washburn, J. Trivedi, L. Catoire, M. Beckstead, Combust. Sci. Technol. 180 (2008) 1502-1517.

[27] Y. Fabignon, J.-F. Trubert, D. Lambert, O. Orlandi, J. Dupays, AIAA Paper 2003-4807 (2003)

[28] B. T. Bojko, P. E. DesJardin, E. B. Washburn, Combust. Flame 161 (2014) 3211-3221

[29] S. Gallier, F. Sibe, O. Orlandi, Proc. Combust. Inst. 33 (2011) 1949-1956.

[30] P. E. DesJardin, J. D. Felske, M. D. Carrara, J. Prop. Power 21 (2005) 478-485.

[31] V. Sarou-Kanian, J.-C. Rifflet, F. Millot, G. Matzen, I. Gökalp, Proc. Combust. Inst. 30 (2005) 2063-2070.

[32] V. Sarou-Kanian, J.-C. Rifflet, F. Millot, E. Véron, T. Sauvage, I. Gökalp, Combust. Sci. Technol. 177 (2005) 2299-2326.

[33] S. Rossi, E. L. Dreizin, C. K. Law, Combust. Sci. Technol. 164 (2001) 209-237.

[34] A. Braconnier, C. Chauveau, F. Halter, S. Gallier, Int. J. Energ. Mat. Chem. Prop. 17 (2018) 111-124.

[35] A. Braconnier, S. Gallier, F. Halter, C. Chauveau, Proc. Combust. Inst. (In Press) (2020)

[36] A. Braconnier, Etude expérimentale de la combustion d'une particule d'aluminium isolée, Ph.D. thesis, University of Orléans (in French), 2020

[37] A. M. Savel'ev, A. M. Starik, Combust. Flame 196 (2018) 223236.

[38] V. A. Babuk, I. N. Dolotkazin, A. A. Glebov, Prop. Explos. Pyrotech. 30 (2005) 281-290.

[39] L. Talbot, R. K. Cheng, R. W. Schefer, D. R. Willis, J. Fluid Mech. 101 (1980) 737-758.

[40] B. Annis, A. Malinauskas, E. Mason, J. Aerosol Sci. 4 (1973) 271-281.

[41] S. Bakanov, V. Roldughin, Aerosol Sci. Technol. 7 (1987) 249255.

[42] M. Naito, T. Yokoyama, K. Hosokawa, K. Nogi, Nanoparticle technology handbook, Elsevier, 2018.
[43] P. Whitmore, J. Aerosol Sci. 12 (1981) 1-9.

[44] M. Marion, Etudes sur la combustion des particules d'aluminium sous pression, Ph.D. thesis, Orléans, 1996.

[45] E. Shpilrain, K. Yakimovich, High Temp.-High Pressures 5 (1973) 191-198.

[46] B. Glorieux, F. Millot, J. Rifflet, Int. J. Thermophys. 23 (2002) 1249-1257.

[47] K. P. Brooks, M. W. Beckstead, J. Prop. Power 11 (1995) 769_ 780.

[48] P. Durand, B. Vieille, H. Lambare, P. Vuillermoz, G. Bouré, P. Steinfeld, F. Godfroy, J. Guéry, AIAA Paper 2000-3864 (2000).

[49] I. Toumi, J. Comput. Phys. 102 (1992) 360-373.

[50] B. Larrouturou, J. Comput. Phys. 95 (1991) 59-84.

[51] M. Salita, J. Prop. Power 7 (1991) 505-512. 